\title{
Can transcatheter closure of atrial septal defect be regarded as a 'standard' procedure?
}

\author{
James L Wilkinson
}

Royal Children's Hospital, Melbourne, Australia

I $\mathrm{N}$ THIS ISSUE OF Cardiology in the Young, BERGER and colleagues ${ }^{1}$ report on 200 patients who had undergone transcatheter closure of interatrial defects with the Amplatzer ${ }^{\text {TM }}$ Septal Occluder. Of these patients, 127 had defects in the oval fossa, 68 had persistence of the oval foramen, and had experienced a presumed paradoxical embolic stroke, and the remaining 5 had undergone fenestration of a Fontan baffle. They raise the question of whether such treatment ". . can be used as standard therapy for closure of atrial septal defects"?

By means of transthoracic echocardiography, they documented complete closure in $95.5 \%$ of their patients after 48 hours, and found only haemodynamically trivial residual shunts in the remainder. By one year, the proportion with any residual shunting had fallen to $1.5 \%$. Mean time required for fluoroscopy was 8.7 minutes, which they regard as being a reflection of the relative ease of implantation of this device compared with alternative systems. ${ }^{2-4}$

This large series is the most substantial report yet to appear of catheter closure of interatrial defects. It certainly offers solid support to previous reports on the use of this device, confirming the simplicity of implantation, the low rates of complications, and the high rate of complete closure of defects.

Some of the limitations and remaining questions concerning the use of devices to close such defects, however, are alluded to only in passing. Several aspects deserve further comment:

- What proportion of patients with an atrial septal defect are suitable for device closure?

- Can the decision be made as to suitability without a transoesophageal echocardiogram? In

Correspondence to: Dr JL Wilkinson, Dept of Cardiology, Royal Children's Hospital, Flemington Rd, Parkville, VIC 3052, Australia. Tel: +61 (3) 9345 5717; Fax: +61 (3) 9345 6001; E-mail: wilkinsj@cryptic.rch.unimelb.edu.au

Accepted for publication 10 June 1999 children, of course, this procedure will usually necessitate a general anaesthetic.

- Does the presence of prosthetic material, such as metal or fabric, in the heart present any longterm threat?

- What risk of endocarditis, if any, remains in children or adults who have undergone this procedure?

- Is this device superior to other systems currently available?

- How does the option of closure using devices compare with the well-established alternative of surgical repair?

\section{What proportion of patients with an atrial septal defect are suitable for device closure?}

The authors of this report claim that 'transcatheter closure was successful in all 200 selected patients'. This is perhaps a slight exaggeration, in that they report 19 additional procedures in which no implant was made, including two patients in whom a device was implanted, but was then removed prior to telease because of instability. Overall, they suggest that more than $90 \%$ of patients initially chosen for closure', and nearly $80 \%$ of 'all unselected patients with an defect in the oval fossa', underwent successful procedures.

The proportion of patients seen during this period who were unsuitable for other reasons, such as defects extending beyond the oval fossa, sinus venosus defects, and atrioventricular septal defects with only atrial shunting, is not stated. There are certainly a number of patients with defects where the margin of the oval fossa is deficient posteriorly, or who elsewhere may have inadequate rims to their defect. It is unclear as to whether such patients are included in the figures given, or were excluded on the grounds that their defects extended beyond the margins of the oval fossa. 
It is noteworthy that 15 patients undergoing successful closure had 'multiple' defects, and five were managed successfully with the use of two devices, implanted at the same procedure, in one instance with the rims of the two devices overlapping one another. This certainly confirms the view that the presence of multiple defects does not exclude the possibility of successful closure. $^{5}$

\section{Can the decision as to suitability be made} without a transoesophageal echocardiogram?

Because of the widely recognised limitations of transthoracic echocardiography, it seems unlikely that a final decision about suitability for closure will be universally achievable without recourse to other modalities of imaging. As transoesophageal echocardiography is necessary for monitoring during the implantation, it is often appropriate in children to defer the final decision about suitability until the time of the procedure itself, unless there are clear anatomic features, demonstrable on transthoracic echocardiography, which preclude the option of interventional closure. In adults, where the limitations of transthoracic echocardiography are more frequently an issue, and in whom transoesophageal echocardiography can usually be carried out under sedation, suitability in most cases may be determined prior to the implantation. In either situation, sizing with balloons, at the time of the procedure, along with intra-operative transoesophageal echocardiography, is the 'final arbiter' on the feasibility of closure. Even then, an attempt at implantation, with the prospect of easy retrieval of the device if it proves to be unstable, may be the ultimate test. There are likely to be a small number of cases, only two in this experience, where a procedural failure occurs after an attempt at insertion of the device.

\section{Does the presence of prosthetic material in the heart present any long-term threat?}

The long-term outlook for patients who have had surgical repair of atrial septal defects is generally extremely good, with minimal, if any, risk of endocarditis. The only significant issue for most patients is the increased risk of atrial arrhythmias in middle adult life. There is some risk of thrombo-embolic events in the early period after patch closure of septal defects, but this is probably not an issue, other than intra-operatively, for patients in whom the defect is repaired by direct suture. The major concerns about the presence of prosthetic material in devices relate to the thrombo-embolic risks and the potential risk of late endocarditis. Thus far, the reported incidence of significant thrombo-embolic problems with the Amplatzer ${ }^{\mathrm{TM}}$ Septal Occluder has been very low, but published experience remains very small.

A small number of transient peri-procedural events, classed as transient ischaemic attacks, have been presented informally at several meetings of investigators, without any reported late sequels. Most operators recommend heparinisation during the procedure, and use anti-platelet therapy for three months after implant, usually confined to Aspirin alone. In this report, Heparin was given intravenously for 48 hours, but this is not employed universally.

Berger and colleagues ${ }^{1}$ report no unexpected arrhythmias, and other published accounts have not referred to significant problems in this respect. We are aware, however, of several patients, mainly adolescents and adults, in whom transient and/or repetitive arrhythmias, such as supraventricular tachycardia, atrial flutter or atrial fibrillation, have occurred in the early days or weeks after implantation. There are no data on the incidence of such late arrhythmias, and so far no adequate data to compare these complications occurring after closure with devices to events occurring after surgical repair.

One theoretical concern about devices that contain Nitinol is that nickel, which is potentially toxic, may gradually elute from the alloy into the circulation, or into tissues adjacent to the implant, and build up to significant levels. Thus far, there is very little evidence to support or to allay such concerns. Studies are under way to try to clarify this matter.

\section{What risk of endocarditis, if any, remains in children or adults who have had this procedure?}

Thus far, there is only one report of endocarditis related to this device, which occurred early after the implantation before endothelialisation would have been expected to be complete. ${ }^{6}$ This event was not related to the procedure as such, and followed a septicaemia which occurred several weeks after closure. No information is currently available to clarify whether there is any ongoing risk of endocarditis after interventional closure of atrial septal defects, but most operators advise appropriate prophylaxis for six months after the procedure, after which it is anticipated that the device will be completely endothelialised. 


\section{Is this device superior to other systems} currently available?

Direct comparisons between the Amplatzer ${ }^{\mathrm{TM}}$ Septal Occluder and other systems, each in the hands of the same operators and over a comparable period of time, are few. ${ }^{2}$ Several advantages have been suggested for the Amplatzer device, including the small size of sheaths, its self-centering properties, the ease of its retrieval if the position or stability of the device are unsatisfactory, and the high rate of complete closure after successful implantation. The low rate of malpositioning and embolisation are also quoted as desirable features of this device. In the series of Berger and colleagues, ${ }^{1}$ one device embolised after implantation, and was successfully retrieved interventionally, obviating the need for surgical removal. Other operators have recorded a small number of successful retrievals of this device following embolisation, and this appears to be a realistic possibility in skilled hands. ${ }^{4}$

Walsh and colleagues ${ }^{3}$ compared the Amplatzer device with the Sideris 'Buttoned' device in two groups of patients who underwent implantation over different periods of time - the Amplatzer device having been adopted because of some concerns about the Sideris device. The fluoroscopy time was significantly shorter with the Amplatzer device, indicative of the much simpler mechanics of implantation, and the rate of complete closure was much higher after one year (93\% versus $44 \%$ ).

There have been several systems introduced in the last five years about which data remains somewhat limited. The so-called 'Angel Wings' device, and the 'ASDOS' system, have been associated with difficulties at implantation, residual shunts, embolisations and other problems. The rate of implantation of these devices appears to have slowed quite markedly in the last year. The Sideris device has been used widely in many countries, but has been abandoned in a number of centres because of the high residual rate of shunting coupled with other problems.

The re-designed 'Clamshell' device, now called 'CardioSEAL' and a closely related 'self-centering' version called 'Starflex', are being used in a substantial number of centres. These devices are relatively easy to implant, though they require rather larger sheaths than does the Amplatzer system. The very flat profile of these devices, which align neatly against the atrial septum, may be an advantage over the bulkier Amplatzer device. The much less substantial metal content of these devices may also be advantageous, as also may be better endothelialisation, though neither of these theoretical advantages is proven. The CardioSEAL device may have specific advantages for multi-fenestrated atrial septal defects, and for defects associated with an aneurysm of the floor of the oval fossa .?

\section{How does the option of interventional closure compare with the well-established alternative of surgical repair?}

Surgery, especially in the current era of smaller and cosmetically less unsightly skin incisions and subsequent scars, offers an attractive, very safe, and highly reliable means of achieving closure of atrial septal defects. There is a large amount of follow-up data, accumulated over more than 40 years. There have been very few late problems, although arrhythmias in middle adult life can be troublesome for a few patients. Concerns about the potentially adverse effects of cardio-pulmonary bypass, peri-operative morbidity, and more prolonged convalescence remain the main points that create momentum towards interventional closure, both for patients and parents and for the treating cardiologists.

At the present time, the lack of any follow-up data in the long term on patients who have had closure with any of the presently available and currently 'in favour' devices remains a source of slight concern.

The lack of such information is the main cause for reluctance to move to full-scale adoption of interventional closure as the preferred option for treatment of interatrial communications in the oval fossa.

It seems reasonable to suggest that transcatheter closure, using the Amplatzer device or other devices such as CardioSEAL or Starflex, should be offered as a non-surgical alternative for patients who prefer to avoid a thoracotomy. It should not be recommended, however, as the procedure of choice for suitable patients until more follow-up information becomes available. Thus, it is probably premature to suggest that Amplatzer device closure "can be used as standard therapy for closure of atrial septal defects" (my italics).

\section{References}

1. Berger F, Ewert P, Björnstad PG, Dahnert I, Krings G, BrillaAustenat I, Vogel M, Lange PE: Transcatheter closure as standard treatment for most interatrial defects: experience in 200 patients treated with the Amplatzer Septal Occluder. Cardiol Young 1999 (9)5: 468-473

2. Formigari R, Santoro G, Rossetti L, Rinelli G, Guccione P, Ballerini L: Comparison of three different atrial septal defect occlusion devices. Am J Cardiol 1998;82: 690-692

3. Walsh KP, Tofeig M, Kitchiner DJ, Peart I, Arnold R: Comparison of the Sideris and Amplatzer septal occlusion devices. Am J Cardiol 1999;83: 933-936 
4. Wilkinson JL, Goh TH: Early clinical experience with use of the 'Amplatzer Septal Occluder' device for atrial septal defect. Cardiol Young 1998;8: 295-302

5. Pedra CA, Fontes-Pedra SR, Esteves CA, Assef J, Fontes VF, Hijazi ZM: Multiple atrial septal defects and patent ductus arteriosus: successful outcome using two Amplatzer septal occluders and Gianturco coils. Cathet Cardiovasc Diagn 1998;45: 257-259
6. Bullock AM, Menahem S, Wilkinson JL: Infective endocarditis on an occluder closing an atrial septal defect. Cardiol Young 1999;9: 65-67

7. Kaulitz R, Paul T, Hausdorf G: Extending the limits of transcatheter closure of atrial septal defects with the double umbrella device (CardioSEAL). Heart 1998;80: 54-59 